\title{
PERIOSTEAL PEDICLE GRAFTING IN DOGS
}

\author{
K. F. King, Melbourne, Australia \\ From the University Department of Surgery and the Orthopaedic Department, \\ St Vincent's Hospital, Melbourne
}

\begin{abstract}
An experimental model was designed to find a theoretical solution to the clinical problem of congenital tibial pseudarthrosis in children. The osteogenic properties of periosteum having been accepted, paired pedicled flaps of periosteum were raised from the subcutaneous surface of the tibia, tubed and anastomosed in a series of young dogs. In the last twenty-four consecutive cases the rate of complete ossification in these tubes rose to one in three. In the later stages of the work, in addition to the construction of periosteal tube grafts, pseudarthroses were induced in the middle third of each canine tibia in order to see if hypertrophy would occur under the stress of weight-bearing. It was found that in each of twenty-four specimens some hypertrophy occurred in the ossified periosteal tube grafts, and in three of the specimens this hypertrophy was associated with complete ossification of the graft that by-passed the pseudarthrosis.
\end{abstract}

The role of periosteum in bone formation has been the subject of many celebrated studies and controversies for more than a century. The work of Ollier, published in 1867 , seemed to prove conclusively that periosteum, divorced from bone, would produce bone in its own right. His results were accepted for over forty years and his beliefs were reinforced by Axhausen's work on bone grafting in 1908. In 1912, however, the publication of Macewen's The Growth of Bone raised serious doubts about established views, as his extensive series of experiments seemed to prove that periosteum had no capacity to produce new bone.

In his Jacksonian Prize Essay of 1917, Hey Groves compared the experimental work of Ollier and of Macewen in great detail. He concluded that periosteum is osteogenic in young animals but only if elevated by sharp dissection, with preservation of a middle or cambium layer lying between bone and periosteum. He also made the point, from his own experimental work, that new bone can be formed either from the periosteum or from the surface of the underlying bone. His final summing-up was that periosteum is chiefly a limiting membrane and that dense bone can live, grow, undergo repair and produce fresh periosteum after the original periosteum has been removed. He believed, however, that in young bones it was possible to elevate the periosteum in such a way as to produce an osteogenic membrane, this probably being due to the lifting of the "epiosteum" (his word) with the periosteum. He felt that in adult bones this was impossible except after trauma or inflammation.

In 1928 Leriche and Policard described how they had repeated Ollier's experiments by raising pedunculated periosteal strips and wrapping them round muscle bellies. Their conclusion was that Ollier was wrong and that if periosteal strips did produce new bone, it was only in cases where microscopic flakes on the deep surface of the membrane had been elevated by sharp dissection-an opinion previously expressed by Davis and Hunnicutt in 1915. The credit for the new bone formation was given entirely to these minute flakes of cortical bone. In their control experiments they raised the periosteum by what they called "blunt dissection", which involved tearing the periosteum off by traction rather than by using a sharp elevator. The fact that such flaps produced no bone was accepted as proof that periosteum possessed no inherent osteogenic property.

When first considered, these experimental results seem to be just as puzzling now as they were to Hey Groves; the key is the importance of preserving an adequate periosteal blood supply. Both Leriche and Hey Groves emphasised that raising periosteum by sharp (and therefore less traumatic) dissection would produce osteogenic flaps, and that the raising of flaps by blunt (rough) dissection would produce mere fibrous tissue. In none of the experiments described by Ollier, Macewen, Davis and Hunnicutt or Leriche was any attempt made to raise periosteal flaps in such a way as to preserve adequate vascular pedicles.

Trueta and Cavadias (1955) were the first to emphasise the relationship between periosteal circulation and osteogenic properties. They observed that proliferation of the periosteal blood vessels was followed by new bone formation in the subperiosteal region. They also noted that if the periosteum was stripped from cortical bone and kept separated from it by a polythene membrane, a large involucrum formed round the whole shaft external to the polythene. In these experiments the periosteal blood supply was carefully preserved.

Various authors have investigated the possibility that free grafts of periosteum might possess osteogenic properties in their own right. Brown and Brown (1913) and

Kevin F. King, F.R.C.S., F.R.A.C.S., Alcaston House, 2 Collins Street, Melbourne, 3000, Victoria, Australia. 
Cohn and Mann (1914) concluded that they did not. Burman and Umansky (1930), Haldeman (1933), De Bruyn and Kabisch (1955), and Cohen and Lacroix (1955) all demonstrated some osteogenic power in free strips of periosteum, and Fell (1932) found that embryonic periosteum in tissue culture could generate bone. Zucman, Maurer and Berbesson (1968) felt that periosteal cells retain osteogenic potential even though temporarily devascularised.

As for clinical surgery, Skoog (1965) has used periosteal flaps raised from the palatal surface of the maxilla to obtain bony closure of palatal defects in children. These flaps were raised by sharp dissection and the published results strongly support the osteogenic properties of well vascularised periosteum.

The problem that prompted the following experiments was that of congenital pseudarthrosis of the tibia, which frequently resists various methods of cortical and cancellous bone grafting. It was while I was examining the possibilities of using periosteum in the treatment of this condition that two hypotheses were investigated.

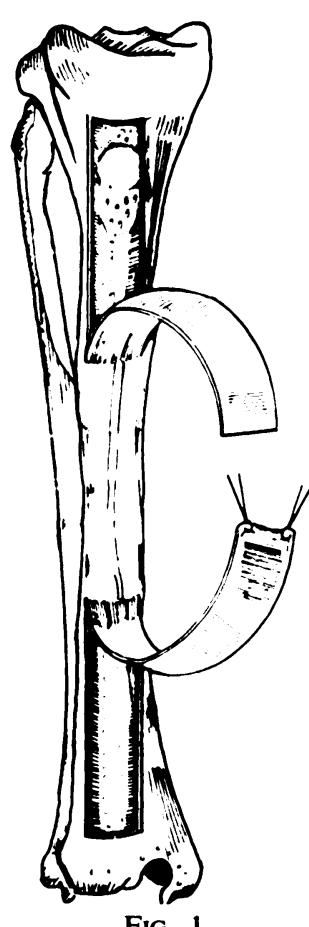

Fig. 1

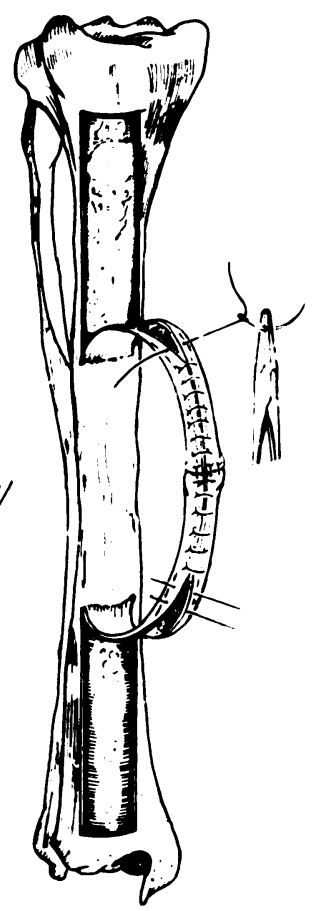

Fig. 2
Figure 1-The first stage of construction of the periosteal tube graft. Proximal and distal flaps have been raised by sharp dissection. Figure 2-The second stage. The two flaps have been tubed and anastomosed round Gelfoam.

1) If two flaps of periosteum are raised from the subcutaneous surface of a tibia in a young animal, with preservation of a blood supply through the base of each flap, and if the free ends are sutured together and the whole rolled in to form a tube, it should be possible to produce a bar of living bone by-passing the middle third of the bone. 2) If a pseudarthrosis is present in the middle third of the tibia, such a bar of living bone may hypertrophy under the stress of weight-bearing.

\section{DETAILS OF THE EXPERIMENTS}

Male and female mongrel puppies aged between six weeks and six months were used. They were kept in indoor kennels at night but allowed to run free in the outdoor exercise yard during the day. One hindleg, usually the right, was used in each puppy.

Anaesthesia-For this purpose each dog was given intravenous pentothal ( 30 milligrams/kilogram body weight) and intubated. Anaesthesia was maintained with $\mathrm{N}_{2} \mathrm{O}$, oxygen and halothane. Operative technique-After careful preparation of the skin, an Esmarch's bandage was applied to the upper thigh without exsanguination; this assured that the periosteal vessels remained partly filled and easily visible. Microsurgical instruments were routinely used in conjunction with Zoiss operating binoculars $(\times 2)$.

A slightly curved longitudinal incision was made just lateral to the subcutaneous border of the tibia, over muscle and extending for the full length of the bone. The subcutaneous surface of the tibia was then exposed without damaging the periosteum or its blood vessels. The adjacent muscles were raised from the anterolateral and posteromedial borders, to expose the periosteal arteries running longitudinally. From this point onwards the steps of each operation depended on the different experiments.

Experiment 1-The construction of periosteal tube grafts based on pedicles-Based on the middle third of the subcutaneous surface of the tibia, periosteal flaps were raised from the proximal and distal thirds using a small sharp periosteal elevator. The periosteum of the middle third was left undisturbed. The medial and lateral margins of each flap were cut to include the periosteal arteries on the anterolateral and posteromedial borders of the tibia. These arteries were clearly visible, not only through the operation loop but also to the naked eye. Each flap was then turned back on itself and the free ends joined with interrupted sutures of 5-0 nylon (Fig. 1). The edges were then rolled in to form a tube, the former deep surfaces facing into the lumen (Fig. 2). In order to prevent the tube from collapsing, a roll of Gelfoam 2 millimetres in diameter and dampened with saline was laid in position, and the edges of the flaps were approximated over it with a continuous 5-0 nylon suture. The length to width ratio of the flaps varied from $3: 1$ to $2: 1$.

The arterial supply of the periosteum imposed strict limits on the design of the flaps and excluded the use of spiral flaps to gain length. The periosteum in these young animals proved to be a tough layer, usually about 0.5 millimetre thick, which could be easily handled and sutured. Each flap was mobilised sufficiently to allow approximation and tubing of the two flaps with the least possible kinking of their bases. The tourniquet was then removed, haemostasis secured and the skin closed with a continuous subcuticular suture of 4-0 chromic catgut. The suture line was covered with a layer of plastic skin from an aerosol container; no other dressing was used. This experiment was performed on forty-two hindlegs in forty-two animals.

Results-The animals were killed at intervals varying from three weeks to six months. Some ossification had developed in every periosteal tube. In the early specimens the bony bridge was usually incomplete at its midpoint, the site of anastomosis between the flaps; in many cases this defect was only obvious on radiographic examination. With gradual improvement in the operative technique the vascular pedicles were more frequently preserved and the rate of complete ossification rose to one in three in the last twenty-four consecutive cases (Fig. 3). Histological cross-sections through the 


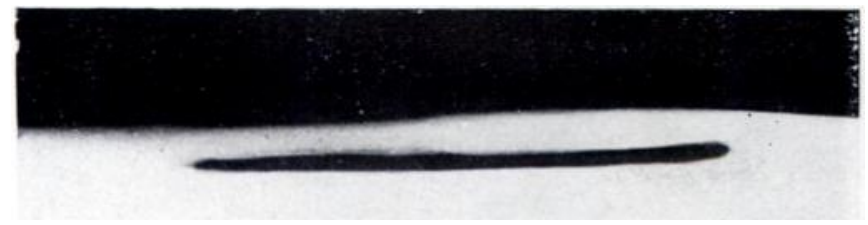

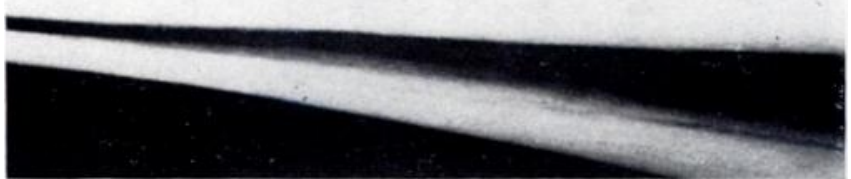

FIG. 3

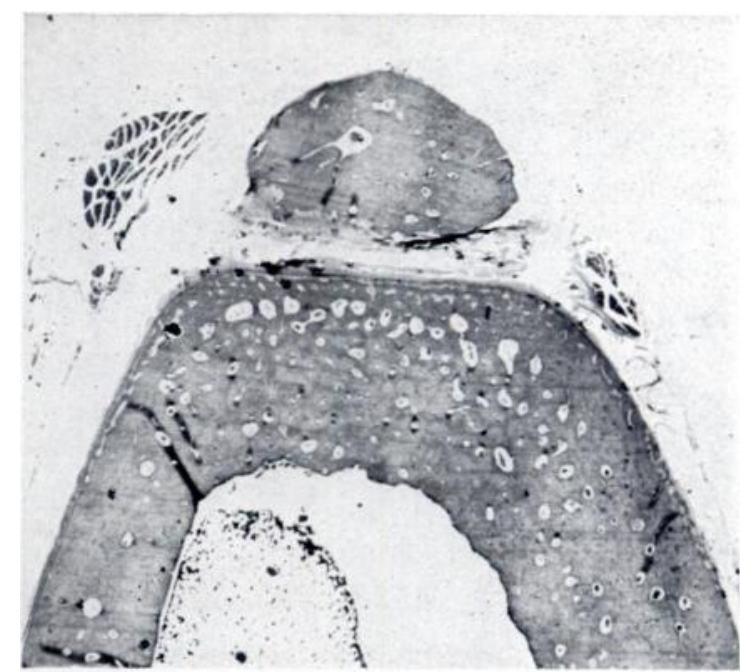

FIG. 4

Figure 3-A lateral radiograph of a completely ossified periosteal tube graft six months after operation $(\times 3$.) Figure 4-A microphotograph of a cross-section from the middle of the same tibia as in Figure 3, showing the ossified graft at the top of the picture $(7$ micron section, $\times 6$.)

tibiae and ossified grafts showed that the periosteal tubes had been replaced by mature woven bone in each specimen (Fig. 4). Experiment 2-The production of a pseudarthrosis in the middle third of the tibia and its effect on a periosteal tube graft-Half a centimetre of bone and periosteum was excised from the middle third of the tibia with a Gigli saw and a periosteal tube graft was constructed. No external splinting was used. This operation was performed on ten tibiae in ten dogs.
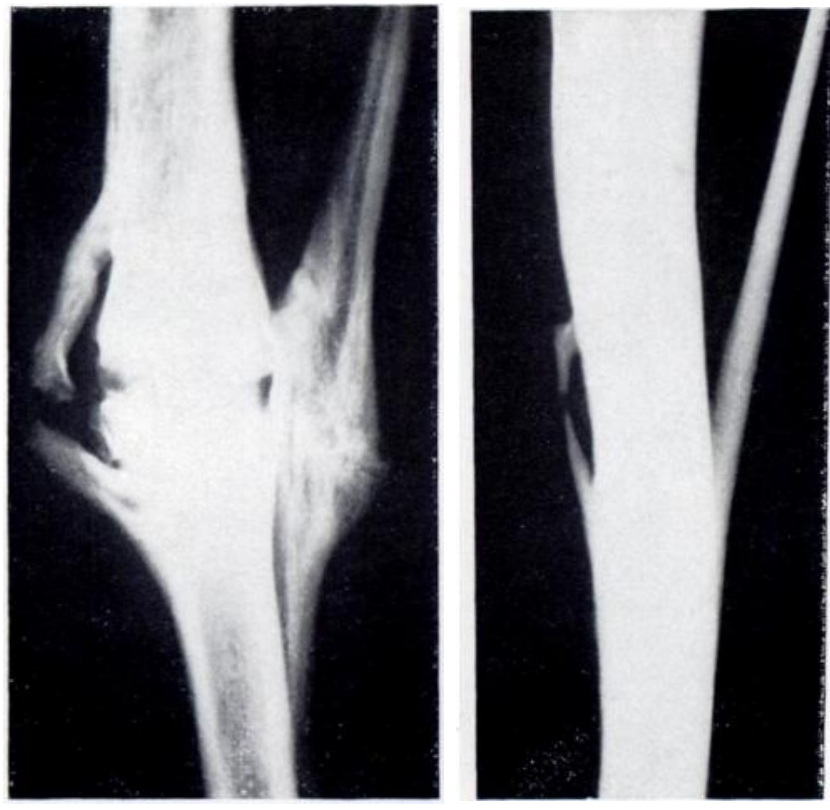

FIG. 5

On the left, an enlarged lateral radiograph taken six months after the making of a pseudarthrosis in the middle third of the tibia, showing the hypertrophy of the incompletely ossified graft. On the right, a comparable radiograph taken six months after the construction of a simple periosteal tube graft. No hypertrophy is present.

Results - The animals were killed at intervals varying from two to six months. It was found that the excised segment had been replaced by bone in nine of the ten specimens. In one specimen, however, there was an established pseudarth- rosis with marked hypertrophy of the ossified periosteal tube graft (Fig. 5).

Experiment 3-The production of a pseudarthrosis in the middle third of the tibia by means of a prosthesis and its effect on a periosteal tube graft-A prosthesis was designed to perform three tasks, firstly, to assure pseudarthrosis formation in the middle third of the tibia; secondly, to give sufficient stability for weight-bearing without external splinting; and thirdly, to allow sufficient movement of the pseudarthrosis to stimulate hypertrophy in the periosteal tube graft. It consisted of a stainless steel intramedullary rod and two perforated metal cups. The rod measured 10 centimetres in length and $\mathbf{0 . 2}$ centimetre in diameter, and was threaded over a distance of 1.5 centimetres at its pointed end. The two stainless steel cups were 1 centimetre in diameter and 0.5 centimetre deep and perforated on their basal surfaces by a hole 0.3 centimetre in diameter, enabling the cups to be threaded over the rod. The rod could be shortened by cutting off a section from the unthreaded end.

Technique-With the minimum of interference to the soft tissues, 0.5 centimetre of bone and periosteum was excised from the middle of the tibia with a Gigli saw and bone nibblers. The two metal cups, their basal surfaces opposed, were placed over the cut ends of the bone. The rod was then drilled down the medullary cavities of the fragments, impaling the cups (Fig. 6). Two periosteal flaps were then raised and tubed as described in Experiment 1. This experiment was performed on twenty-four tibiae in twenty-four animals. Results-The animals were killed at intervals varying from four to six months. The findings in the tube grafts varied from slender incomplete bridges up to massively hypertrophied bars of bone (Fig. 7). Complete ossification with hypertrophy occurred in three animals (Figs. 8 to 10).

\section{DISCUSSION}

This study shows that periosteum is more than the enveloping membrane described by Macewen and that its properties are of potential use to the clinician.

Young dogs were chosen as the experimental animals for several reasons. Periosteum in puppies is a relatively tough membrane, it is easy to handle surgically providing 


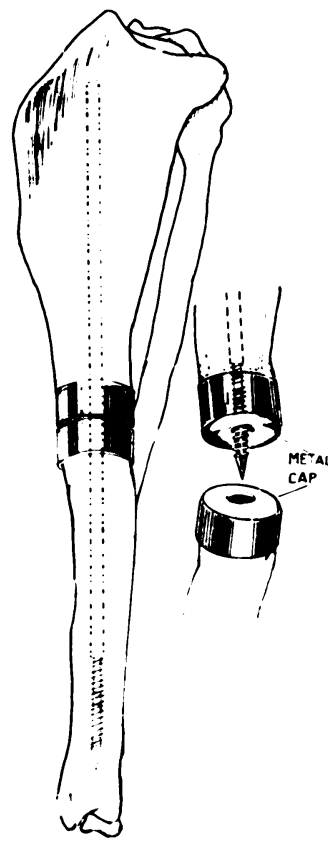

Fig. 6

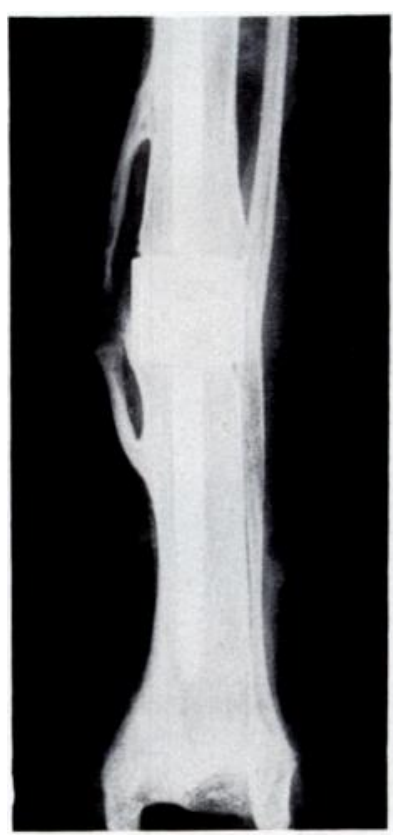

Fig. 7

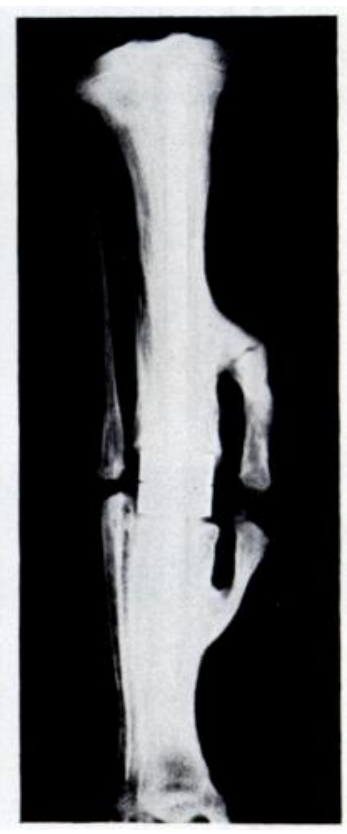

Figure 6-A diagram to show the technique of insertion of the prosthesis. Figure 7On the left, a radiograph taken four months after insertion of a prosthesis and construction of a periosteal tube graft. The graft has ossified incompletely and has not hypertrophied. On the right, a comparable radiograph taken five months after insertion of the prosthesis and construction of a periosteal tube graft. The graft shows marked hypertrophy.

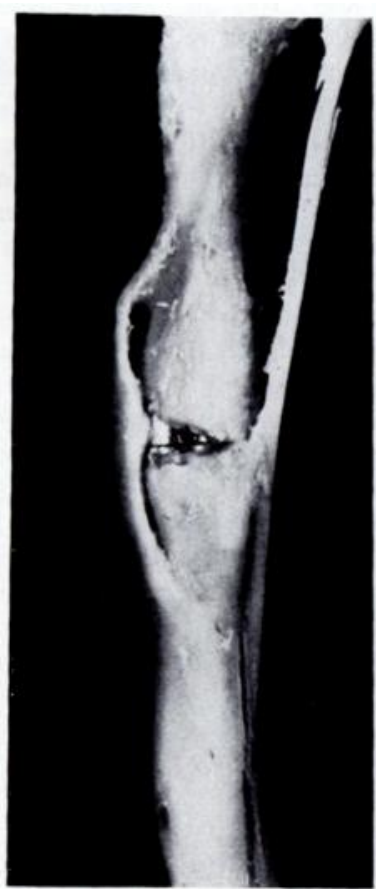

FiG. 8

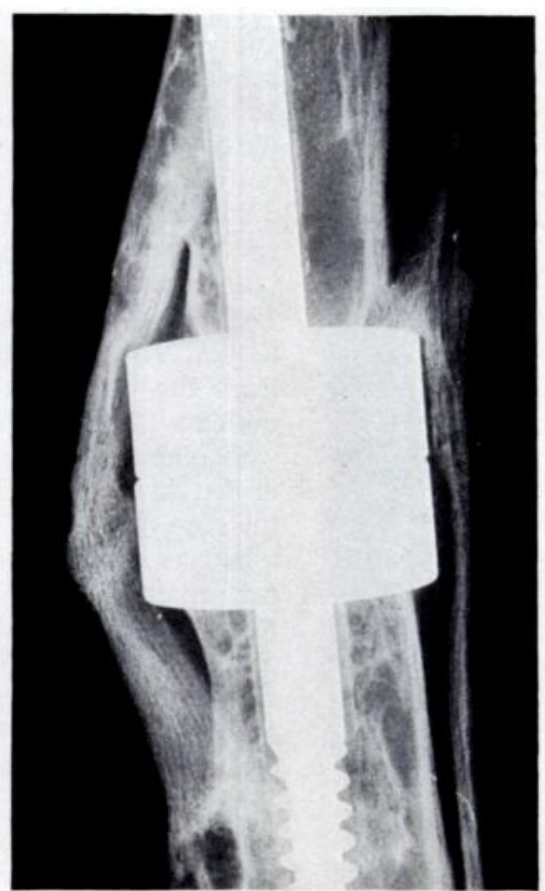

Fig. 9

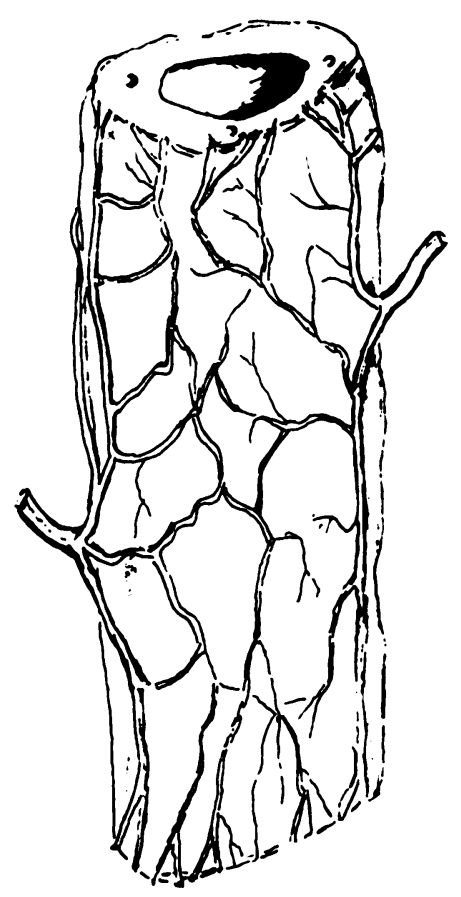

Fig. 10

Figure 8-A specimen of a completely ossified periosteal tube graft obtained six months after operation. In the presence of a pseudarthrosis the graft has hypertrophied. Figure 9-A lateral radiograph obtained four months after construction of a pseudarthrosis. The periosteal tube graft has ossified completely and has hypertrophied. Figure 10-A drawing of a dissected specimen showing the periosteal blood supply on the subcutaneous surface of the tibia. $(\because 3$. 
fine instruments are used, and thus easy to fashion into flaps and tubes. An important feature is that its blood supply is very similar to that of human periosteum, consisting of longitudinal arteries, one for each of the three tibial borders. These vessels extend the whole length of the bone, and numerous side branches form fine networks on each of the three main tibial surfaces. The main feeding artery to this periosteal circulation joins it in the middle third of the bone.

In these experiments the arguments as to whether the osteogenic properties of the flaps were due to the "epiosteal" layer (Hey Groves), to microscopic bone flakes (Leriche) or to the inherent capacity of well vascularised periosteum (Trueta) were avoided by using young animals and sharp dissection to raise the flaps. As one of the objects was to see if a well vascularised periosteal tube would produce a bar of bone, the exact layer of the periosteum involved was not important, as long as bone was produced.

In Experiment 1 the early results were encouraging although the bridges were rarely complete. When the pedicles were more carefully based on the vascular pattern the incidence of complete ossification rose markedly. The fact that it did not rise higher than one in three was probably due to the inherent vascular complications associated with raising flaps and tubes of skin, namely oedema and kinking, thrombosis and stretching of nutrient vessels. In Experiment 3 the small number of three out of twenty-four grafts which combined hypertrophy with complete ossification was only to be expected, taking into account the inevitable disturbance of the periosteal blood supply associated with insertion of the prosthesis.

These experiments show that the behaviour of periosteal flaps is predictable provided they retain an adequate blood supply. In our present state of knowledge the findings seem to be divorced from clinical practice, especially as in the usual orthopaedic operation periosteum tends to be handled with relative roughness and with scant respect for its intrinsic blood supply. However, the periosteal blood vessels can be clearly seen with the naked eye, and more so with operating binoculars. The standard methods of autogenous bone grafting, while satisfactory in most situations, do have the disadvantage that most of the bone cells die during transfer, due to inevitable devascularisation. The most promising feature of the periosteal pedicle graft is that the flap remains viable and so retains its capacity for osteogenesis. With the modern tools now available, particularly the instruments of micro-surgery and micro-angiography, periosteum may well change from being a relatively neglected tissue to one of great practical importance. It is possible that orthopaedic surgeons may one day employ viable periosteal flaps to obliterate bone defects with the confidence and facility displayed by plastic surgeons when using skin flaps and tubes in the more superficial parts of the body.

I wish to acknowledge the advice and assistance of Professor R. Bennett, Department of Surgery, St Vincent`s Hospital, Melbourne.

\section{REFERENCES}

Axhausen, G. (1908) Die histologischen und klinischen Gesetze der freien Osteoplastik auf Grund von Thierversuchen. Archiv für klinische Chirurgie, 88, 23-145.

Brown, W. L., and Brown, C. P. (1913) Preliminary report on experimental bone and periosteal transplantation. Surgery, Gynecology' and Obstetrics, 17, 681-689.

Burman, M. S., and Umansky, M. (1930) An experimental study of free periosteal transplants, wrapped around tendon. Journal of Bone and Joint Surgery, 12, 579-594.

Cohen, J., and Lacroix, P. (1955) Bone and cartilage formation by periosteum. Journal of Bone and Joint Surgery, 37-A, 717-730.

Cohn, I., and Mann, G. (1914) The osteogenic function of periosteum and bone transplants: Research. Southern Medical Journal, 7, $214-218$.

Davis, J. S., and Hunnicutt, J. A. (1915) The osteogenic power of periosteum: with a note on bone transplantation. Johns Hopkins Hospital Bulletin, 26, 69-82.

De Bruyn, P. P. H., and Kabisch, W. T. (1955) Bone formation by fresh and frozen, autogenous and homogenous transplants of bone, bone marrow and periosteum. American Journal of Anatomy, 96, 375-405.

Fell, H. B. (1932) The osteogenic capacity in vitro of periosteum and endosteum isolated from the limb skeleton of fowl embryos and young chicks. Journal of Anatomy, 66, 157-180.

Groves, E. W. H. (1917) Methods and results of transplantation of bone in the repair of defects caused by injury or disease. British Journal of Surgery, 5, 185-242.

Haldeman, K. O. (1933) The influence of periosteum on the survival of bone grafts. Journal of Bone and Joint Surgery, 15, $302-319$.

Leriche, R., and Policard, A. (1928) The Normal and Pathological Physiology of Bone: Its Problems. St Louis: C. V. Mosby Company.

Macewen, W. (1912) The Growth of Bone. Glasgow: James Maclehose and Sons.

Ollier, L. (1867) Traité expérimental et clinique de la régénération des os et de la production artificielle du tissu osseux. 2 vols. Paris: Victor Masson et Fils.

Skoog, T. (1965) The use of periosteal flaps in the repair of clefts of the primary palate. Cleft Palate Journal, 2, $332-339$.

Trueta, J., and Cavadias, A. X. (1955) Vascular changes caused by the Küntscher type of nailing. Journal of Bone and Joint Surgery, 37-B, 492-505.

Zucman, J., Maurer, P., and Berbesson, C. (1968) The effect of autografts of bone and periosteum in recent diaphysial fractures. Journal of Bone and Joint Surgery, 50-B, 409-422. 